\title{
Comprometimento Organizacional: Bases para uma Abordagem Processual ${ }^{1}$
}

\author{
Fabíola Marinho Costa ${ }^{2}$ \\ Universidade Federal do Recôncavo da Bahia \\ Antonio Virgílio Bittencourt Bastos \\ Universidade Federal da Bahia
}

\begin{abstract}
RESUMO - Há uma importante vertente de pesquisa sobre comprometimento organizacional (CO) sobre os vínculos entre indivíduo e sua organização empregadora. O presente estudo objetiva caracterizar a produção científica que analisa esse construto em uma perspectiva processual, pela sua importância para esclarecer questões conceituais e empíricas. Foram analisados39 artigos que relatam pesquisa longitudinal sobre CO, publicados entre 1975 e 2010. Além de constatar a reduzida utilização deste desenho de pesquisa na área, verificou-se: a) que o foco central dos estudos é a identificação dos seus antecedentes, mais do que nas mudanças no comprometimento ao longo do tempo e seus fatores determinantes; b) são reduzidos os estudos que incorporam variáveis do contexto. Da revisão são extraídas algumas recomendações para estudos futuros.
\end{abstract}

Palavras-chave: comprometimento organizacional, estudos longitudinais, contextualização em estudos organizacionais

\section{Organizational Commitment: Basis for a Process Approach}

\begin{abstract}
The links between individuals and their employer organization have been a main focus within research on organizational commitment (CO). The present study aimed to characterize the scientific literature analyzing this construct from a process perspective, considering its importance in clarifying conceptual and empirical issues. 39articles reporting longitudinal data on CO, published between 1975 and 2010, were analyzed. In addition to confirming the infrequent use of this research design in the area, the analysis pointed out that: a) a central focus on the identification of its antecedents, more than on changes in commitment patterns over time and their determining factors; b) only a reduced number of studies incorporate context variables. This review results in relevant recommendations for future studies.
\end{abstract}

Keywords: organizational commitment, longitudinal studies, contextualizing organizational research

Ao longo das últimas décadas fortaleceu-se uma compreensão de que fenômenos sociais, psicossociais, psicológicos, quer no nível dos indivíduos, quer dos grupos ou unidades sociais maiores, são dinâmicos, fluidos, complexos por dependerem de uma rede de fatores em interação. Esse pressuposto também está presente quando se analisa o mundo do trabalho e, mais especificamente, os vínculos de compromissos desenvolvidos entre o trabalhador e a organização empregadora, que passam a ser vistos como algo que é construído processualmente e que se altera ao longo do tempo. No entanto, pouca atenção tem sido dada à questão de como esse fenômeno muda com o tempo (Beck \& Wilson, 2000, 2001), sendo essa considerada uma questão crítica para compreender o curso da relação entre o trabalhador e sua organização (Bentein, Vandenberg, Vandenberghe, \& Stinglhamber, 2005) e para nos aproximarmos dos fatores efetivamente causais envolvidos na construção e mudança do comprometimento do trabalhador com a sua organização.

Essa lacuna torna-se mais surpreendente quando se verifica que a literatura organizacional é fortemente dominada pelo tema da mudança - nas tecnologias, nos processos de trabalho, nos modelos de organização, nos modelos de

1 Apoio: $\mathrm{CNPq}$

2 Endereço para correspondência: Centro de Ciências da Saúde (CCS), Universidade Federal do Recôncavo da Bahia (UFRB), Avenida Carlos Amaral, 1015, Cajueiro, CEP: 44570-000, Santo Antonio de Jesus, Bahia, Brasil.E-mail: fabiolamcosta@gmail.com gestão, no perfil dos trabalhadores, para ficarmos em apenas algumas das suas dimensões mais centrais. As relações entre mudanças no mundo do trabalho e comprometimento é um dos desafios para a agenda de pesquisa da área (Bastos, 1998; Meyer, 2009), podendo ser a porta de entrada para uma efetiva abordagem processual do fenômeno.

Ao se discutir o que seria uma abordagem processual do comprometimento, aqui entendida como aquela que asseguraria capturar as mudanças no fenômeno e os fatores que as afetam, três linhas de reflexão são importantes. A primeira consiste na inclusão nas pesquisas, do contexto maior (organizacional e societal) em que o comprometimento é investigado, (quando, por exemplo, comprometimento é estudado em conjunto com as intensas mudanças que estão reconfigurando o mundo do trabalho e, por extensão, as organizações); a segunda linha de reflexão implica a retomada das discussões teóricas e conceituais sobre a própria natureza do comprometimento (seu caráter mais disposicional ou contextual e, em decorrência, a ênfase na estabilidade versus mudança do comprometimento). Finalmente, o terceiro eixo é de caráter metodológico e envolve a análise das contribuições das pesquisas de delineamento longitudinais, pelo que esses desenhos permitem capturar de alterações no fenômeno estudado, introduzindo a dimensão temporal como crítica para identificar processos e assegurar maior segurança sobre os seus potenciais fatores determinantes.

O presente texto estrutura-se em três segmentos que buscam sistematizar as contribuições teóricas e empíricas sobre 
os três eixos acima apontados, associados a uma abordagem processual do comprometimento organizacional. O presente estudo inicia sua exposição discorrendo sobre a contextualização da pesquisa em comportamento organizacional. Em seguida, apresenta considerações teóricas sobre as definições do comprometimento e sua natureza desse fenômeno e, por fim, apresenta uma revisão da pesquisa sobre comprometimento organizacional conduzida a partir de delineamentos longitudinais.

\section{Comportamento Organizacional: o Desafio de uma Perspectiva Contextual e Processual}

Entende-se o contexto como atributos do sistema físico e social onde o indivíduo se insere (Nord \& Fox, 2004). O termo contexto vem de uma raiz latina que significa "fazer uma ligação" e contextualizar, portanto, requer ligar observações a um conjunto de fatos, eventos e pontos de vista relevantes (Rousseau \& Fried, 2001). Já os principais fatores disposicionais são as características de personalidade (o mais estável de todos os elementos disposicionais), os motivos e as atitudes, sendo que estas últimas não são necessariamente estáveis, podendo variar a partir de eventos cotidianos (Cunha, Rego, Cunha, \& Cabral-Cardoso, 2004). As pessoas podem apresentar uma atitude em um contexto e outra em outro ou, ainda, mudar de acordo com alguma experiência pessoal (Gouveia, Santos, \& Milfort, 2009).

A importância do contexto para a compreensão de quaisquer produtos ou resultados organizacionais é enfatizada por Peiró e Tetrick (2011). Os autores diferenciam seis diferentes âmbitos de análise que vão do individual, passam pelo conteúdo do trabalho, pelas equipes ou grupos e chegam a três âmbitos contextuais mais amplos: um societário (fatores sociais, econômicos, políticos, nacionais ou internacionais); um segundo âmbito envolve as interfaces entre trabalho e não trabalho (conflito família-trabalho, desemprego, sono etc.); e um terceiro o nível organizacional (clima, políticas e modelos de gestão). Temos, assim, um vasto leque de fatores contextuais que podem jogar significativo papel tanto nos processos organizacionais, quanto nas formas de intervir sobre eles.

A contextualização é mais importante na pesquisa do comportamento organizacional contemporâneo do que foi no passado por duas razões: 1) o domínio da pesquisa organizacional está se tornando mais internacional, aumentando os desafios da transposição de modelos das ciências sociais de uma sociedade para a outra; e 2) a natureza do trabalho e dos ambientes de trabalho, em rápido processo de diversificação, pode alterar substancialmente a dinâmica causal subjacente às relações trabalhador-organização (Rousseau \& Fried, 2001). Nessa perspectiva, é necessário explorar e desenvolver caminhos alternativos para contextualizar os fenômenos investigados nas pesquisas organizacionais.

Colocar o contexto em foco abre possibilidades de se atentar para relações que tornam todos os fenômenos humanos processuais. O comprometimento organizacional, como um vínculo, sempre foi visto como produto de relações entre o indivíduo e a organização e, neste sentido, a pesquisa deveria sempre estar atenta para os elementos contextuais. Os modelos explicativos de comprometimento, consolidados em algumas metanálises (Cooper-Hakin \& Vieswesvaran, 2005; Meyer, Stanley, Herscovitch \& Topolnytsky, 2002; Riglle, Edmondson, \& Hansen, 2009) e sinteticamente apresentado por Bastos, Rodrigues, Moscon, Silva e Pinho (2013) têm se sofisticado na identificação de múltiplas variáveis com poder preditivo, envolvendo desde características pessoais, passando por características do gestor, do grupo, do trabalho e da organização. No entanto, constata-se, claramente, que entre os preditores distais, predominam variáveis pessoais e organizacionais. Variáveis do contexto macro (social, econômico, político, tecnológico) estão praticamente ausentes da pesquisa sobre comprometimento.

A pesquisa sobre antecedentes ou preditores de comprometimento concentra-se fortemente em variáveis proximais relacionadas a características do trabalho, às relações com a liderança e em percepções de características ou políticas organizacionais (suporte, percepção de justiça) e às condições do papel desempenhado pelo sujeito.

Em síntese o contexto mais amplo (tanto organizacional, quanto societal), quando incluídos nas pesquisas sobre comprometimento, o são a partir de elementos discretos, isolados e sem considerar a sua dinâmica, apesar do acúmulo de reflexões e evidências das transformações em curso no mundo do trabalho e, em decorrência, nas organizações, quer nos seus modelos estruturais, quer nos seus modelos de gestão.

Assim, as implicações das mudanças no mundo do trabalho na natureza das relações entre trabalhador e organização são consideradas questões básicas para futuras pesquisas em comprometimento organizacional (Becker, Klein, \& Meyer, 2009). Assim como, é necessário conhecer mais sobre o desenvolvimento do comprometimento e seu impacto nas mudanças, visto que é importante considerar tanto as mudanças no mundo do trabalho, como as mudanças que ocorrem nos trabalhadores (Becker et al., 2009). Nesse sentido, ao vincular o estudo do comprometimento ao estudo das mudanças abre-se a possibilidade de analisá-lo sob uma perspectiva processual. Essas mudanças podem ser percebidas pelos trabalhadores como violações do contrato psicológico, levando a fortes reações afetivas (Rousseau, 1995), assim como podem causar um sentimento de falta de justiça organizacional, afetando negativamente o comprometimento organizacional (Meyer, 2009).

As mudanças em curso no mundo do trabalho causaram forte impacto nas pesquisas da área do Comportamento Organizacional (Rousseau, 1997). Pôde-se observar um deslocamento da ênfase dos estudos organizacionais de uma visão dos indivíduos, independentemente de seu contexto, para a consideração da interação entre os indivíduos e seus contextos organizacionais e sociais (Nord \& Fox, 2004). É possível afirmar, no entanto, que os estudos organizacionais continuam ainda clivados entre os níveis micro e macro (Cunha et al., 2004), apesar dos esforços e da consciência de que devam ser articulados. Embora as dificuldades metodológicas inerentes a esse empreendimento possam explicar as reduzidas tentativas de investigação que articulam diferentes níveis de análise, não se justifica que o contexto continue sendo analisado predominantemente como um agregado de fatores estáticos que descrevem a organização na percepção dos próprios indivíduos. 


\section{O Construto Comprometimento: suas Definições e sua Natureza}

Apesar da significativa trajetória histórica como objeto de pesquisa que se intensificou a partir dos anos 1980, pode-se afirmar que ainda não existe um consenso sobre a definição que melhor caracterize o comprometimento organizacional, sendo esse um construto complexo e polissêmico (Menezes \& Bastos, 2009).

Algumas definições sobre o comprometimento, como bem destacam Klein, Molloy e Cooper (2009), possibilitam uma confusão entre esse e alguns de seus antecedentes (a exemplo de investimentos e trocas, identificação, congruência de objetivos e valores), ou consequentes (a exemplo de motivação e continuação). Para os autores, há três perspectivas de definir comprometimento que procuram não confundi-lo com outros fenômenos: comprometimento como uma atitude (attitude); comprometimento como uma força (force); e comprometimento como um vínculo (bond). A Tabela 1 sintetiza os principais argumentos dos autores ao distinguirem as três perspectivas conceituais sobre comprometimento. et al., 2009). Como um exemplo, pode-se citar a definição apresentada por Meyer (2009), na qual o comprometimento é uma força interna que vincula um indivíduo a um objeto (social ou não social) e/ou a um curso de ação de relevância para esse objeto.

Na perspectiva conceitual de comprometimento como um vínculo, proposta por Klein et al. (2009), esse seria definido como o apego psicológico de um indivíduo a uma organização. Dentro de uma perspectiva psicológica, os autores defendem que tratar de percepções é mais adequado do que de força ou pressão e que definir comprometimento como um vínculo percebido possibilita um melhor reconhecimento do papel da cognição social.

Definido como um vínculo, o comprometimento pode ser visto como um vínculo em geral ou como um tipo particular de vínculo (Klein et al., 2009). A primeira visão envolve uma definição mais ampliada, a exemplo do modelo tridimensional de Meyer e Allen $(1991,1997)$ que trata as três diferentes bases todas como comprometimento. Já a segunda visão considera que há diferentes tipos de vínculos

Tabela 1. Perspectivas conceituais sobre comprometimento. Fonte: Klein et al. (2009)

\begin{tabular}{|c|c|c|c|}
\hline Natureza & Definição & Autores & Questões \\
\hline ATITUDE & $\begin{array}{l}\text { Comprometimento envolve } \\
\text { sentimentos como apego, identificação } \\
\text { ou lealdade com o objeto de } \\
\text { compromisso. }\end{array}$ & $\begin{array}{l}\text { Mowday, Porter \& Steers } \\
\text { (1982), Meyer \& Allen } \\
\text { (1991), Morrow (1993) }\end{array}$ & $\begin{array}{l}\text { Modelo tripartide X perspectiva } \\
\text { cognitivista. }\end{array}$ \\
\hline FORÇA & $\begin{array}{l}\text { Comprometimento é uma força } \\
\text { interna que vincula um indivíduo a } \\
\text { um objeto (social ou não social) e/ou } \\
\text { a um curso de ação de relevância para } \\
\text { esse objeto. }\end{array}$ & Meyer (2009) & $\begin{array}{l}\text { Forças distintas (emoções, cognições, } \\
\text { pressões grupais e culturais) podem } \\
\text { conduzir a mesmos comportamentos. } \\
\text { Expansão indevida do conceito } \\
\text { de comprometimento, com a } \\
\text { pulverização de bases. }\end{array}$ \\
\hline VÍNCULO & $\begin{array}{l}\text { Comprometimento como um estado } \\
\text { psicológico que reflete o quão } \\
\text { fortemente uma pessoa está ligada a } \\
\text { um foco de compromisso. }\end{array}$ & Klein et al. (2009) & $\begin{array}{l}\text { Um vínculo geral } \mathrm{X} \text { tipo específico de } \\
\text { vínculo. }\end{array}$ \\
\hline
\end{tabular}

A conceituação de comprometimento como uma atitude acarreta questionamentos levantados na literatura psicológica a respeito da visão tripartite de atitudes (uma justaposição afeto-comportamento-cognição), que vem sendo suplantada por uma visão de atitude como um sumário de avaliação (atribuindo dimensões como: bom-mau, prejudicial-benéfico, agradável-desagradável) acerca do objeto psicológico em foco (Klein et al., 2009). Ou seja, atitudes representam uma integração avaliativa de cognições e afetos experimentados em relação a um objeto, sendo julgamentos avaliativos que integram e sumarizam reações cognitivas e afetivas (Crano \& Prislin, 2006). Ser comprometido com um objeto é diferente de um breve julgamento do quão favorável ou desfavorável ele é considerado, e que não é preciso ter uma visão favorável de um objeto para ser comprometido com esse. Nesse sentido, a conceituação de comprometimento como uma atitude não é a mais apropriada descrição do construto (Klein et al., 2009).

Por meio da conceituação de comprometimento como uma força, defende-se que os antecedentes do comprometimento criam pressões que ligam o indivíduo a um objeto, mantendo um comportamento direcionado a esse (Klein percebidos e que nem todos podem ser caracterizados como comprometimento. Nessa perspectiva, a identificação e o consentimento (compliance), por exemplo, são considerados vínculos distintos do comprometimento (Klein et al., 2009). O primeiro refere-se a um vínculo marcado por uma fusão psicológica do eu com o objeto em foco (Klein et al., 2009) e o segundo refere-se a um vínculo marcado pela aceitação da influência de outra pessoa ou grupo com o objetivo de alcançar uma resposta favorável (Kelman, 2006).

A perspectiva que considerada o comprometimento como um vínculo em geral conduziu a modelos multidimensionais como o proposto por Meyer e Allen (1991), em que diferentes processos psicológicos gerariam a mesma atitude (comprometimento), o que foi sintetizado no conceito de diferentes bases do comprometimento (afetiva, continuação e normativa). As críticas a esse modelo vêm se acumulando ao longo dos últimos anos (Osigweh, 1989; Cooper-Hakim \& Viswesvaran, 2005; Solinger, Van Olffen \& Roe, 2008), fortalecendo um movimento de retorno a uma definição unidimensional de comprometimento. As críticas, de uma 
forma geral, apontam que o modelo tridimensional proposto e desenvolvido por Meyer e Allen (1991) constitui, na realidade, um modelo explicativo da permanência na organização, envolvendo um alargamento indevido do conceito de comprometimento.

Estudos desenvolvidos no Brasil (Rodrigues \& Bastos, 2012; Scheible, Bastos, \& Rodrigues, 2007; Silva \& Bastos, 2010) apontam caminhos e evidências empíricas bastante fortes para afirmar que as diferentes bases propostas por Meyer e Allen (1991) são na realidade distintos vínculos do indivíduo com a organização (gerados por antecedentes distintos e com impactos diferentes no comportamento no trabalho). A proposta dos construtos "entrincheiramento organizacional" (Rodrigues \& Bastos, 2012) e "consentimento organizacional” (Silva \& Bastos, 2010) revelam a tendência de se considerar comprometimento um vínculo específico e definido a partir da identificação e dos afetos dirigidos ao objeto.

Em meio à discussão acerca da conceituação do comprometimento (atitude, força ou vínculo) e sua natureza (uni ou multidimensional), apresenta-se como importante a problematização do comprometimento como algo estático ou dinâmico. É importante reconhecer que, qualquer que seja a perspectiva, o conceito de comprometimento busca explicar a consistência do comportamento ou a relativa estabilidade do comportamento do trabalhador. Ele é um construto voltado para explicar permanência, manutenção de índices adequados de produtividade, qualidade de desempenho, entre outras características desejáveis ou esperadas pelas organizações.

O crescimento da pesquisa sobre comprometimento, que superou temas clássicos como motivação e satisfação, é creditado, em parte, ao fato de ele ser uma característica mais estável (Mowday, Porter, \& Steers, 1982). As atitudes são passíveis de mudança, mas essas mudanças não são fáceis e rápidas, especialmente quando a força do componente afetivo é grande e ela envolve um conjunto rico de crenças ou cognições. Por outro lado, as atitudes são mais passíveis de mudança em função da experiência do que os traços de personalidade, que seriam disposições mais rígidas e estruturantes do indivíduo.

Isto não significa, entretanto, que o comprometimento (como atitude, força ou vínculo) seja algo estático e imune às mudanças nos próprios indivíduos ou nos contextos em que se inserem. No entanto, se, por um lado, parte dos teóricos defende que o comprometimento é um processo que se altera através do tempo e, portanto, dinâmico, por outro lado, os pesquisadores tendem a examiná-lo como um construto estático (Becker et al., 2009), em parte em função do largo predomínio de desenhos de pesquisa transversais que envolvem apenas uma única mensuração do fenômeno.

A possibilidade de tratar o comprometimento como um vínculo, permite capturar melhor a natureza processual e dinâmica deste fenômeno que é essencialmente algo relacional e não apenas do indivíduo. Compromisso implica sempre um objeto e, quase sempre, a percepção de reciprocidade, troca, relação. Tomar o comprometimento como um vínculo permite analisar as relações estabelecidas entre o trabalhador e sua organização, o que vem requerendo a incorporação da questão do contexto e das mudanças e, portanto, uma perspectiva processual e dinâmica do fenômeno.
No próximo segmento, vamos nos deter na caracterização das pesquisas longitudinais envolvendo comprometimento buscando-se avaliar, em que medida, contexto e mudanças têm sido incorporados de modo a assegurar uma visão mais processual do comprometimento.

\section{Comprometimento Organizacional: o que nos dizem os estudos de corte longitudinal}

Os estudiosos organizacionais têm lentamente incorporado o fenômeno temporal dentro das pesquisas organizacionais (Roe, Waller, \& Clegg, 2009). Esses estudos respondem ao que Riketta e Van Dick (2009) descrevem como uma notável carência de estudos longitudinais que possibilitem análises para responder questões de causalidade.

Muitas teorias nas ciências organizacionais são de natureza longitudinal, com suas variáveis subjacentes descritas em termos dinâmicos (Ployhart \& Vandenberg, 2010). Entretanto, a grande maioria de estudiosos dessas teorias ainda utiliza, em suas pesquisas, desenhos de corte transversal, considerando somente um único ponto no tempo, por meio dos quais são feitas inferências de associações entre variáveis estáticas. A variabilidade associada a um construto, em um dado tempo, pode ser completamente diferente da variabilidade associada ao construto através do tempo e, portanto, examinar a mudança de uma variável ao longo do tempo demanda o uso de desenhos de pesquisa longitudinal (Ployhart \& Vandenberg, 2010).

A enorme carência, apesar do crescimento lento de estudos longitudinais, também caracteriza a área dos estudos sobre comprometimento organizacional (Wright \& Kehoe, 2009). Dentre as mais importantes questões a serem discutidas em pesquisas da área está a relativa ao desenvolvimento do comprometimento (Becker et al., 2009). A compreensão de como o comprometimento se desenvolve e de quais fatores estão relacionados às mudanças no comprometimento auxilia no preenchimento de lacunas do trabalho empírico e no fortalecimento da teoria em comprometimento (Beck \& Wilson, 2000, 2001).

A análise do desenvolvimento do comprometimento, entretanto, pode envolver dois processos que, embora próximos, não são idênticos: 1) processo de formação do comprometimento; e 2) processo de mudança de comprometimento. A discussão de como se forma o comprometimento apresenta-se dominante, incluindo o exame de variáveis antecedentes associadas ao fenômeno. Já a análise do processo de mudança do comprometimento envolve uma perspectiva mais dinâmica do fenômeno. Como já mencionado, ainda que haja um reconhecimento por parte dos teóricos de que o comprometimento é um processo que se desenvolve através do tempo, os pesquisadores tendem a examiná-lo como um construto estático (Becker et al., 2009), dando pouca atenção à questão de como o comprometimento muda com o tempo (Beck \& Wilson, 2000, 2001).

Desde o clássico trabalho de Mowday et al. (1982), já são encontradas bases para uma abordagem processual do comprometimento, que foi negligenciada pela grande maioria da pesquisa que se produziu a seguir. Esses autores enfocam os diferentes fatores que podem influenciar o comprometimento 
do trabalhador, em vários estágios da carreira, desde o período da escolha do trabalho, passando pelas experiências iniciais de socialização, até a estabilização e o entrincheiramento. Segundo os autores, os indivíduos entram na organização com diferentes propensões para se tornarem comprometidos, em função de características pessoais (valores, crenças, personalidade), expectativas sobre o trabalho e circunstâncias associadas à decisão de se vincular à organização. Durante os primeiros meses de emprego, fatores como características do trabalho, supervisão, grupos de trabalho e pagamento, formam as experiências relacionadas ao trabalho que têm grande influência sobre o comprometimento (Mowday et al., 1982). Por fim, são consideradas as influências não relacionadas à organização, como a avaliação de alternativas de emprego. Nessa perspectiva, o comprometimento parece se desenvolver lentamente e constantemente através do tempo, de modo que eventos transitórios não deveriam causar uma séria reavaliação do empregado acerca do seu vínculo com a organização.

Diante do exposto, torna-se relevante a realização de pesquisas em uma perspectiva longitudinal e que considerem o contexto. Essa conjunção possibilita a investigação da mudança do comprometimento ao longo do tempo nos indivíduos, vistos em interação com um ambiente potencialmente impactante. Diversos estudiosos da área do comprometimento vêm enfatizando tanto a necessidade de se considerarem as mudanças no mundo do trabalho (e.g. Becker et al., 2009; Cohen, 2003; Meyer \& Allen, 1997) quanto à importância do desenvolvimento de estudos longitudinais (e.g. Becker et al., 2009; Cohen, 2003; Cohen \& Freund, 2005; Mathieu \& Zajac, 1990; Meyer et al., 2002; Mowday et al., 1982; Wright \& Kehoe, 2009).

É importante que pesquisadores identifiquem eventos históricos, como mudanças em políticas e estruturas, que podem afetar o comprometimento dos trabalhadores (Beck \& Wilson, 2001). Atualmente, as organizações são raramente estáticas e, com frequência, vêm passando por mudanças nos processos, procedimentos, sistemas e estruturas. Em graus variados, tanto o contexto organizacional quanto o contexto relacionado ao ambiente no qual a organização está inserida exercem alguma influência no processo por meio do qual o comprometimento se desenvolve (Beck \& Wilson, 2001).

Um primeiro trabalho de revisão dos estudos longitudinais foi desenvolvido por Morrow (2011). O foco da revisão realizada voltou-se para identificar fatores preditores de comprometimento, no sentido de fornecer elementos para a gestão do comprometimento pelas organizações, ou seja, o interesse central foi o de ampliar a compreensão de como gestores podem, de forma proposital e deliberada, aumentar o comprometimento dos empregados. Práticas de socialização, mudanças organizacionais, práticas de recursos humanos, relações interpessoais e relações empregado-organização têm sido apontados, em pesquisas com desenhos longitudinais, como antecedentes do comprometimento organizacional afetivo (Morrow, 2011).

Diferente da revisão de Morrow (2011), algumas questões norteiam a presente revisão. Ao longo de quase três décadas de pesquisa empírica, em que medida os estudos longitudinais contribuíram para a compreensão da dinâmica do comprometimento, tomando-o como um processo e não como uma característica estática dos indivíduos? Em que medida os estudos longitudinais incorporaram variáveis do contexto macrossocial no sentido de explorar possíveis determinantes para a dinâmica do comprometimento? Buscando evidências para essas questões, realizamos um levantamento e análise de um conjunto de trabalhos de pesquisa caracterizados por serem de corte longitudinal.

Para identificar os artigos que relatavam pesquisas com desenho longitudinal sobre comprometimento organizacional, foram, inicialmente, selecionadas revistas internacionais, disponíveis no Portal de Periódicos da Capes (Coordenação de Aperfeiçoamento de Pessoal de Nível Superior), que possuíam pelo menos um artigo sobre comprometimento organizacional, no período de 2004 a 2010. Foram identificados 28 periódicos, todos internacionais. Nesses periódicos, foi examinada toda a produção sobre comprometimento organizacional, da qual foram extraídos os trabalhos que classificados como estudos longitudinais e que se transformaram no corpus que fundamentou a presente revisão. Nesse levantamento, a seleção dos artigos obedeceu aos seguintes critérios: as publicações deveriam ter, de forma clara, a variável comprometimento organizacional como objeto de estudo; os estudos deveriam apresentar corte longitudinal, definido por envolver a mensuração de variáveis organizacionais em pelo menos dois momentos temporais distintos. Também foram incluídos artigos citados pelos artigos identificados nas revistas relacionadas e que obedeciam aos critérios, ampliando a amostra para artigos desde o ano de 1975, atingindo-se um total de 51 referências. Há dois artigos publicados nos anos 1970; 10 nos anos 1980; 19 nos anos 1990 e 20 artigos publicados a partir de 2000. Diante do volume de pesquisa empírica sobre comprometimento, verifica-se, desde já, o pequeno peso das pesquisas longitudinais no conjunto total de publicações. Este reduzido número de pesquisas longitudinais é atribuído, por Morrow (2011), às dificuldades de participação das organizações em múltiplas coletas de dados e as pressões institucionais para que os pesquisadores publiquem estudos com frequência.

A análise apresentada neste segmento do trabalho, no entanto, limita-se a 39 artigos a que se teve acesso ao texto completo, eliminando-se os casos de acesso apenas ao abstract, pela impossibilidade de acesso a informações centrais para a presente análise. Essa análise envolveu o levantamento, em cada artigo, dos seguintes aspectos: a) abordagem de comprometimento utilizada; b) instrumento usado para mensurar o comprometimento; c) número de medidas do fenômeno ao longo do tempo; d) principais resultados, aqui explorados em termos da dinâmica do comprometimento. A Tabela 2 sintetiza os principais resultados obtidos na análise dos artigos.

A primeira dimensão analisada no conjunto de artigos reporta-se à abordagem adotada em relação ao comprometimento organizacional, explorando-se o peso da abordagem unidimensional, representada pela clássica proposta de mensuração de Mowday et al. (1982) em relação à abordagem tridimensional de Meyer e Allen (1991). Embora conceitualmente possamos entender o comprometimento como uma força, um vínculo ou uma atitude (Klein et al., 2009), na pesquisa empírica o construto é operacionalizado sempre como uma atitude. Todos os trabalhos utilizam es- 
Tabela 2. Características dos artigos longitudinais sobre comprometimento organizacional.

\begin{tabular}{|c|c|c|}
\hline Dimensões analisadas & Categorias & $\begin{array}{l}\text { Número de artigos / } \\
\text { frequência }(\%)\end{array}$ \\
\hline \multirow{6}{*}{$\begin{array}{l}\text { Abordagem do } \\
\text { comprometimento }\end{array}$} & Unidimensional (escala de Mowday et al. - OCQ) & $18(46,2)$ \\
\hline & $\begin{array}{l}\text { Unidimensional (escala de comprometimento afetivo de Meyer \& } \\
\text { Allen) }\end{array}$ & $4(10,2)$ \\
\hline & Multidimensional (escala de Meyer \& Allen) & $8(20,5)$ \\
\hline & $\begin{array}{l}\text { Ambas (escala de Mowday et al. - OCQ; escala de Meyer \& } \\
\text { Allen) }\end{array}$ & $4(10,2)$ \\
\hline & Outras escalas & $5(12,8)$ \\
\hline & Ausência de referencia ao contexto & $21(53,8)$ \\
\hline \multirow{3}{*}{$\begin{array}{l}\text { Inserção de elementos } \\
\text { do contexto na pesquisa }\end{array}$} & $\begin{array}{l}\text { Simples caracterização geral do contexto onde a pesquisa foi } \\
\text { realizada ou dos participantes }\end{array}$ & $8(20,5)$ \\
\hline & $\begin{array}{l}\text { Inclusão de variáveis do contexto organizacional como preditores } \\
\text { de mudança no comprometimento }\end{array}$ & $10(25,7)$ \\
\hline & Inclusão de variáveis do contexto macrossocial & $0(0)$ \\
\hline \multirow{4}{*}{$\begin{array}{l}\text { Número de medidas de } \\
\text { comprometimento no } \\
\text { tempo }\end{array}$} & Apenas uma medida & $10(25,7)$ \\
\hline & Duas medidas & $15(38,5)$ \\
\hline & Três medidas & $8(20,5)$ \\
\hline & Mais de três medidas & $6(15,4)$ \\
\hline \multirow{3}{*}{ Resultados obtidos } & Foco no desenvolvimento (antecedentes) & $22(56,4)$ \\
\hline & Foco na mudança (alterações) & $3(7,7)$ \\
\hline & Ambos (antecedentes e mudança) & $14(35,9)$ \\
\hline \multirow{5}{*}{$\begin{array}{l}\text { Dinâmica do } \\
\text { comprometimento }\end{array}$} & Crescimento & $2(5,1)$ \\
\hline & Declínio ao longo do tempo & $9(23,1)$ \\
\hline & Estabilidade & $3(7,7)$ \\
\hline & Crescimento e/ou decréscimo entre os tempos & $3(7,7)$ \\
\hline & Não se aplica & $22(56,4)$ \\
\hline
\end{tabular}

calas atitudinais, modelo Likert, com destaque para a escala OCQ (Organizational Commitment Questionaire) proposta por Mowday et al. (1982), que toma o construto como uma estrutura unidimensional e que foi utilizada em 46,2\% dos trabalhos. O modelo proposto por Meyer e Allen (1991) vem em seguida, presente em 30,7\% dos artigos, sendo que parte dos trabalhos que usam as escalas propostas por esses autores se limitam a usar a escala do comprometimento afetivo apenas, desconsiderando as dimensões normativa e de continuação. Em síntese, percebe-se que na grande maioria dos estudos, o comprometimento é tratado como um vínculo afetivo e mensurado como uma atitude que incorpora, adicionalmente, crenças sobre o objeto e disposições comportamentais.

Uma segunda dimensão da análise realizada refere-se a como o contexto é tratado nas pesquisas. A inclusão do contexto, especialmente o macrossocial, permitiria tratar o comprometimento como um fenômeno mais processual já que a mudança é um elemento central do mundo do trabalho atual. Os resultados obtidos, em linhas gerais, revelam o quanto o contexto é negligenciado nos estudos empíricos da área. A maioria dos trabalhos não faz referência ao contexto em que o estudo foi realizado $(53,8 \%)$. Oito estudos fazem pequenas descrições dos participantes ou das organizações estudadas, o que não significa que variáveis contextuais tenham sido consideradas nos modelos explicativos desenvolvidos. Os estudos, ao longo da descrição da amostra, identificam o país, o tipo de organização e o tipo de ocupação dos trabalhadores participantes, apresentando, portanto, uma muito breve caracterização do contexto da pesquisa. Em apenas 10 trabalhos $(25,7 \%)$ variáveis do contexto organizacional foram efetivamente incorporadas à pesquisa. São exemplos: processo de fusão entre organizações (Armstrong-Stassen, Cameron, Mantler, \& Horsburgh, 2001), processo de downsizing (Armstrong-Stassen, 2002), processo de mentoria (Donaldson, Ensher, \& Grant-Vallone, 2000), movimentação na carreira (Johnston, Griffeth, Burton, \& Carson, 1993). No conjunto de estudos analisados não se encontrou qualquer caso em que o contexto macrossocial fosse incorporado para ajudar a compreender a dinâmica do comprometimento organizacional. Embora Nord e Fox (2004) apontem que a busca da interação entre os indivíduos e seus contextos organizacionais seja uma tendência geral dos estudos organizacionais, os resultados aqui encontrados revelam que no estudo do comprometimento organizacional, o contexto aparece de forma limitada. 
A terceira dimensão analisada refere-se ao número de medidas envolvidas na pesquisa de forma a caracterizar o estudo como longitudinal. Como se vê na Tabela 2, em 25,7\% dos artigos, embora a pesquisa tenha envolvido medidas em mais de um tempo, a variável comprometimento só foi mensurada uma só vez. Ou seja, são estudos longitudinais, mas que não oferecem uma perspectiva longitudinal sobre comprometimento. Essa medida insere-se em redes de preditores e ou consequentes de outros fenômenos estudados. O padrão mais frequente é medir o comprometimento duas $(38,5 \%)$ ou três vezes $(20,5 \%)$. Encontrou-se um artigo em que comprometimento foi medido seis vezes (Van Maanen, 1975). Há uma grande variabilidade nos intervalos em que as medidas foram realizadas. Assim, mesmo neste conjunto restrito de trabalhos de caráter longitudinal, parte deles sequer oferece uma visão longitudinal do comprometimento organizacional.

A quarta dimensão refere-se aos resultados obtidos nas pesquisas, mais especificamente ao foco do trabalho: identificar antecedentes/preditores que apontam como o comprometimento se desenvolve ou identificar mudanças nos níveis de comprometimento ao longo do tempo e que fatores as explicam. Apoiados na distinção feita por Ployhart e Vandenberg (2010) entre 1) mudança que ocorre dentro de uma unidade e 2) mudança que ocorre entre unidades, buscamos identificar, no caso do comprometimento se o foco da pesquisa era na 1) mudança que ocorre dentro do próprio comprometimento; e/ou 2) na mudança que ocorre envolvendo o comprometimento e outros fenômenos.

Como vemos na Tabela 2 , mais da metade $(56,4 \%)$ dos trabalhos buscam explicar o desenvolvimento do comprometimento e não as mudanças. São pesquisas que utilizam desenhos longitudinais para ampliar as evidências de que alguns fenômenos são efetivamente preditores de comprometimento. Três trabalhos focalizam a mudança no comprometimento, sem investigar fatores explicativos e 35,9\% dos trabalhos articulam ambos os objetivos - identificar a mudança e os seus preditores.

Pode-se afirmar que, em geral, há uma tendência entre os estudos sobre comprometimento a enfatizar sua formação, a partir do estudo de antecedentes como: propensão do indivíduo ao comprometimento, experiências de trabalho iniciais, comprometimento com a ocupação, táticas de socialização do empregado, mentoria de alta qualidade e adaptação à cultura organizacional. Esses resultados são congruentes com o diagnóstico de Beck e Wilson (2001) de que poucas pesquisas têm focado diretamente e explicitamente na identificação de mudanças no comprometimento ao longo do tempo ou nos fatores associados a essas mudanças. As pesquisas tendem a excluir a influência de variáveis macro, como organizacionais ou culturais, no desenvolvimento do comprometimento (Becker et al., 2009).

Finalmente, no conjunto de artigos que oferecem resultados sobre a dinâmica/mudança do comprometimento, identificou-se que tendência foi encontrada ao longo das medidas efetuadas. Os resultados mostram que em apenas dois estudos o comprometimento dos trabalhadores aumenta com o passar do tempo; três estudos encontram certa estabilidade; e nove trabalhos mostram um declínio do comprometimento à medida que o tempo passa. $\mathrm{O}$ padrão de mudança se revela mais complexo em alguns casos, em que há crescimento e depois declínio, ou crescimento seguido de estabilidade. A quantidade de estudos que permitem identificar a mudança é tão reduzida que não nos permite identificar que fatores podem fazer o comprometimento crescer ou decrescer ao longo do tempo. No entanto, dentre os estudos, as mudanças do comprometimento tendem a estar mais relacionadas às experiências no trabalho do que a características individuais. Em alguns estudos, essas experiências são impactadas por variáveis de contexto macro como downsizing (Armstrong-Stassen, 2002), fusão (Armstrong-Stassen et al., 2001), mudanças tecnológicas (Majchrzak \& Cotton, 1988), aquisição (Newman \& Krzystofiak, 1993), e resultam no decréscimo do comprometimento organizacional. Os poucos dados encontrados mostram uma maior tendência ao declínio, apontando que as políticas e práticas organizacionais não estão conseguindo manter os níveis iniciais ou a predisposição dos trabalhadores em se comprometerem com as suas organizações empregadoras.

\section{Considerações Finais}

O presente estudo nasceu da constatação de que, embora a teoria sobre comprometimento organizacional o trate como um fenômeno dinâmico e que muda com o tempo, há uma visível desproporção entre os estudos de corte transversal e os de corte longitudinal na área. Ou seja, a pesquisa empírica, ao medir comprometimento apenas em um tempo, deixa de identificar mudanças ao longo do tempo e, portanto, deixa de explorar que fatores são críticos para essas mudanças. Como sabemos, os estudos longitudinais oferecem maior segurança no estabelecimento de relações de causalidade. Adicionalmente, há uma crescente consciência de que as organizações são unidades socialmente construídas e, portanto, fluidas e dinâmicas, o que deveria estimular mais estudos que considerem tanto os indivíduos quanto as organizações em diferentes momentos do tempo. Finalmente, todo o campo de Estudos Organizacionais tem se direcionado para incorporar às suas pesquisas e teorias o contexto macro social, cultural, econômico e político, pelos impactos que causam nos processos individuais, grupais e organizacionais. Esse fato implica em incorporar nas pesquisas variáveis contextuais, pois é da interação entre elas e os processos individuais que se constituem os processos organizacionais.

Do conjunto de estudos longitudinais analisados é possível extrair algumas conclusões que embasam uma proposta de agenda para os estudos sobre comprometimento organizacional. A pesquisa sobre comprometimento organizacional ainda debate-se com problemas conceituais que se traduzem em abordagens distintas e que fazem com que o conceito tenha uma extensão muito diferente entre os autores. O debate entre perspectivas uni e multidimensionais é presente, atual e está longe de ser superado. Cresce a tendência de considerar o comprometimento um vínculo de natureza afetiva que envolve identificação e apego, distinto de um vínculo calculativo ou instrumental pautado nas perdas em sair da organização. Os estudos longitudinais, como vimos, utilizam ambas as abordagens, o que dificulta a integração dos seus resultados. 
A pesquisa, mesmo longitudinal, ainda é mais voltada para identificar preditores ou consequentes do comprometimento, mais do que para a descrição das mudanças e identificação dos seus determinantes. Ou seja, o desenho longitudinal é utilizado mais para refinar modelos explicativos do que para explorar a dinâmica do comprometimento. Os estudos voltados para entender essa dinâmica, no geral, partem da análise do impacto de processos que estão ocorrendo no nível organizacional (fusão, downsizing etc), o que já significa um avanço na articulação entre os níveis individual e organizacional. Trata-se de uma vertente a ser fortalecida em novas pesquisas, embora ainda não incorporem fenômenos do macro contexto societal.

Diante dessas constatações, é possível identificar como uma tendência a busca por uma definição de comprometimento mais clara e precisa, considerando-o como um vínculo exclusivamente afetivo. Entender o comprometimento como um vínculo apresenta-se como uma possibilidade de analisar relações entre partes, ou seja, relações entre o trabalhador e sua organização. Com isto, pode-se incorporar a questão do contexto e das mudanças, tanto organizacionais quanto relativas ao ambiente sociocultural no qual organizações e trabalhadores estão inseridos. E, finalmente, fazer com que os estudos longitudinais se concentrem em compreender as mudanças que ocorrem nos níveis de comprometimento, fazendo com que fatores individuais, grupais, organizacionais e societais interajam para tornar alguns trabalhadores cada vez mais comprometidos com a sua organização e outros cada vez menos comprometidos.

O reduzido número de estudos longitudinais e a ênfase no desenvolvimento e não na mudança, nos levam a concordar com Becker et al. (2009) de que os pesquisadores tendem a examinar o comprometimento como um construto estático, como uma característica pessoal ou algo que resulta de relações no interior das organizações, desconsiderando o contexto mais amplo em que elas se inserem. Assim, a pesquisa sobre comprometimento encontra-se frente a um duplo desafio: incorporar o contexto (e as suas mutações constantes); ampliar o uso de delineamentos longitudinais que permitam acompanhar possíveis alterações quer no vínculo, quer no contexto.

\section{Referências}

Armstrong-Stassen, M. (2002). Designated redundant but escaping lay-off: A special group of lay-off survivors. Journal of Occupational and Organizational Psychology, 75, 1-13.

Armstrong-Stassen, M., Cameron, S. J., Mantler, J., \& Horsburgh, M. E. (2001). The impact of hospital amalgamation on the job attitudes of nurses. Canadian Journal of Administrative Sciences, 18(3), 149-162.

Bastos, A. V. B. (1998, setembro). Comprometimento no trabalho: contextos em mudança e os rumos da pesquisa deste domínio [Tape recording]. In Anais, 22. Encontro da ANPAD, 1998, Foz do Iguaçu, PR.
Bastos, A. V. B., Rodrigues, A. C. A., Moscon, D. C. B., Silva, E. E. C., \& Pinho, A. P. M. (2013). Comprometimento no Trabalho: fundamentos para a gestão de Pessoas. In L. O. Borges, \& L. Mourão (Eds.), O Trabalho e as Organizações - atuações a partir da Psicologia (pp. 279-310). Porto Alegre: Artmed.

Beck, K., \& Wilson, C. (2000). Development of Affective Organizational Commitment: A Cross-Sequential Examination of Change with Tenure. Journal of Vocational Behavior, 56, 114-136.

Beck, K., \& Wilson, C. (2001). Have we studied, should we study, and can we study the development of commitment? Methodological issues and the developmental study of workrelated commitment. Human Resource Management Review, 11, 257-278.

Becker, T. E., Klein, H. J., \& Meyer, J. P. (2009). Commitment in organizations: accumulated wisdom and new directions. In H. J. Klein, T. E. Becker, \& J. P. Meyer (Eds.), Commitment in Organizations. Accumulated Wisdom and New Directions. (pp. 419-452). New York / London: Routledge - Taylor \& Francis Group.

Bentein, K., Vandenberg, R., Vandenberghe, C., \& Stinglhamber, F. (2005). The role of change in the relationship between commitment and turnover: a latent growth modeling approach. Journal of Applied Psychology, 90(3), 468-482.

Cohen, A. (2003). Multiple commitments in workplaces: an integrative approach. Mahwah, New Jersey: Lawrence Erlbaum Associates.

Cohen, A., \& Freund, A. (2005). A longitudinal analysis of the relationship between multiple commitments and withdrawal cognitions. Scandinavian Journal of Management, 21, 329351.

Cooper-Hakim, A., \& Viswesvaran, C. (2005). The construct of work commitment: testing an integrative framework. Psychological Bulletin, 131(2), 241-259.

Crano, W. D., \& Prislin, R. (2006). Attitudes and persuasion. Annual Review of Psychology, 57, 354-374.

Cunha, M. P., Rego, A., Cunha, R. C., \& Cabral-Cardoso, C. (2004). Comportamento organizacional: disposições, situações e percepções (2004). In M. P. Cunha, A. Rego, R. C. Cunha, \& C. Cabral-Cardoso (Eds.), Manual de comportamento organizacional e gestão (pp. 95-120). Lisboa: Editora RH.

Donaldson, S. I., Ensher, E. A., \& Grant-Vallone, E. J. (2000). Longitudinal Examination of Mentoring Relationships on Organizational Commitment and Citizenship Behavior. Journal of Career Development, 26(4), 233-249.

Gouveia, V. V., Santos, W. S., \& Milfort, T. L. (2009). O uso da estatística na avaliação psicológica: comentários e considerações práticas. In C. S. Hutz (Ed.). Avanços e polêmicas em avaliação psicológica: em homenagem a Jurema Alcides Cunha (pp. 127-156). São Paulo: Casa do Psicólogo.

Johnston, M. W., Griffeth, R. W., Burton, S., \& Carson, P. P. (1993). An exploratory investigation in to the relationships between promotion and turnover: A quasi-experimental longitudinal study. Journal of Management, 19, 33-49.

Kelman, H. C. (2006). Interests, relationships, identities: three central issues for individuals and groups in negotiating their social environment. Annual Review of Psychology, 57(1), 01-26. 
Klein, H. J., Molloy, J. C., \& Cooper, J. T. (2009). Conceptual foundations: construct definitions and theoretical representations of workplace commitments. In T. E. Becker, H. J. Klein, \& J. P. Meyer, (Eds.), Commitment in Organizations. Accumulated Wisdom and New Directions (pp. 3-36). New York / London: Routledge - Taylor \& Francis Group.

Majchrzak, A., \& Cotton, J. (1988). A longitudinal study of adjustment to technological change: From mass to computerautomated batch production. Journal of Occupational Psychology, 61, 43-66.

Mathieu, J. E., \& Zajac, D. M. (1990). A Review and MetaAnalysis of the Antecedents, Correlates, and Consequences of Organizational Commitment. Psychological Bulletin, 108(2), 171-194.

Menezes, I. G., \& Bastos, A. V. B. (2009). Bases sociológicas, antropológicas e psicológicas do comprometimento organizacional. Psicologia em Revista, 15(3), 200-215.

Meyer, J. P. (2009). Commitment in a changing world of work. In T. E. Becker, H. J. Klein, \& J. P. Meyer, (Eds.), Commitment in Organizations. Accumulated Wisdom and New Directions (pp. 37-68). New York / London: Routledge-Taylor \& Francis Group.

Meyer, J. P., \& Allen, N. J. (1991). A three-component conceptualization of organizational commitment. Human Resource Management Review, 1, 61-89.

Meyer, J. P., \& Allen, N. J. (1997). Commitment in the workplace: theory, research and application. Thousands Oaks: SAGE.

Meyer, J. P., Stanley, J. D., Herscovitch, L., \& Topolnytsky, L. (2002). Affective, Continuance, and Normative Commitment to the Organization: A Meta-analysis of Antecedents, Correlates, and Consequences. Journal of Vocational Behavior, 61, 20-52.

Morrow, P. C. (1993). The theory and measurement of work commitment. Greenwich, CT: Jai.

Morrow, P. C. (2011). Managing organizational commitment: Insights from longitudinal research. Journal of Vocational Behavior, 79, 18-35.

Mowday, R. T., Porter, L. W., \& Steers, R. M. (1982). Employeeorganization linkages - the psychology of commitment, absenteeism, and turnover. New York: Academic Press.

Newman, J. M., \& Krzystofiak, F. J. (1993). Changes in employee attitudes after an acquisition. Group \& Organizational Studies, 18(4), 390-410.

Nord, W. R., \& Fox, S. (2004). O indivíduo nos estudos organizacionais: o grande ato de desaparecimento? In: S. R. Clegg, C. Hardy, \& W. Nord (Eds.). Handbook de Estudos Organizacionais (pp. 186-225). São Paulo: Atlas.

Osigweh, C. A. B. (1989). Concept fallibility in organizational science. Academy of Management. The Academy of Management Review, 14(4), 579-594.

Peiró, J. M., \& Tetrick, L. (2011). Occupational Health Psychology. In P. R. Martin, F. M. Cheung, M. C. Knowles, M. C. Kyrios, L. Littlefield, J. B. Overmier, \& J. M. Prieto (Eds.), IAAP Handbook of Applied Psychology (pp. 292-315). Oxford: Blackwell Publishing.

Ployhart, R. E., \& Vandenberg, R. J. (2010). Longitudinal research: the theory, design, and analysis of change. Journal of Management, 36(1), 94-120.
Riketta, M., \& Van Dick, R. (2009). Commitment's Place in the Literature. In T. E. Becker, H. J. Klein, \& J. P. Meyer, (Eds.), Commitment in Organizations. Accumulated Wisdom and New Directions (pp. 67-95). New York / London: Routledge - Taylor \& Francis Group.

Riggle, R. J., Edmondson, D. R., \& Hansen, J. D. (2009). A metaanalysis of the relationship between perceived organizational support and job outcomes: 20 years of research. Journal of Business Research, 62, 1027-1030.

Rodrigues, A. C. A., \& Bastos, A. V. B. (2012). Entrincheiramento organizacional: construção e validação da escala. Psicologia: Reflexão e Crítica, 25, 688-700.

Roe, R. A., Waller, M. J., \& Clegg, S. R. (2009). Time in organizational research. New York; London: Routledge.

Rousseau, D. M. (1995). Psychological contract in organizations: understanding written and unwritten agreements. Thousand Oaks, CA: Sage, London.

Rousseau, D. M. (1997). Organizational behavior in the new era. Annual Review of Psychology, 48, 515-546.

Rousseau, D. M., \& Fried, Y. (2001). Location, location, location: contextualizing organizational research. Journal of Organizational Behavior, 22, 1-13.

Scheible, A. C. F., Bastos, A. V. B., \& Rodrigues, A. C. A. (2007, setembro). Comprometimento e Entrincheiramento: Integrar ou Reconstruir? Uma Exploração das Relações entre estes Construtos à luz do Desempenho. [Tape recording]. In Anais, 31. Encontro da ANPAD, 2007, Rio de Janeiro, RJ.

Silva E. E. C., \& Bastos, A. V. B. (2010). A escala de consentimento organizacional: construção e evidências de sua validade. Revista Psicologia: Organizações e Trabalho, 10(1), 7-22.

Solinger, O. N., Van Olffen, W., \& Roe, R. A. (2008). Beyond the three-component model of organizational commitment. Journal of Applied Psychology, 93(1), 70-83.

Van Maanen, J. (1975). Police socialization: A longitudinal examination of job attitudes in an urban police department. Administrative Science Quarterly, 20, 207-228.

Wright, P. M., \& Kehoe, R. R. (2009). Organizational-Level Antecedents and Consequences of Commitment. In T. E. Becker, H. J. Klein, \& J. P. Meyer, (Eds.), Commitment in Organizations. Accumulated Wisdom and New Directions. (pp. 285-307). New York / London: Routledge - Taylor \& Francis Group.

Recebido em 15.10.2011

Primeira decisão editorial em 13.11.2013

Versão final em 17.02.2014

Aceito em 21.03.2014 